\section{G382(P) MIND THE GAP! ELEVATED ANIONS SECONDARY TO PARACETAMOL AND SEPSIS}

'S Hulley, ${ }^{2}$ J Perring, ${ }^{3} \mathrm{~N}$ Manning, ${ }^{3} \mathrm{~S}$ Olpin, 'S Yap. ${ }^{1}$ Metabolic Medicine, Sheffield Children's Hospital, Sheffield, UK; 'Paediatric Intensive Care, Sheffield Children's Hospital, Sheffield, UK; ${ }^{3}$ Clinical Chemistry, Sheffield Children's Hospital, Sheffield, UK

10.1136/archdischild-2015-308599.337

Aim Metabolic acidosis is a common finding in children presenting with sepsis. Hypovolaemia and hypoxia are the common causes for this derangement but sometimes there are other culprits. We aim to highlight the significance of correlating the anion gap with the biochemical picture and, when there are discrepancies, look for alterative diagnoses. An unusual case of transient pyroglutamic aciduria, presenting during an episode of severe sepsis and paracetamol use, will be used to outline the importance of examining the anion gap.

Methods We illustrate the case of a 15 month old girl who presented with an 11 day history of diarrhoea and vomiting. She presented to the emergency department in a state of decreased consciousness. She was found to be hypotensive, hypoglycaemic and have a profound metabolic acidosis. She required mechanical ventilation and fluid resuscitation. Despite these interventions, she continued to have a profound metabolic acidosis with a very high anion gap (30.5). The levels of lactate and ketones were insufficient to explain the clinical picture.

Results Metabolic investigations for the child were instigated. Whilst a majority of these were normal, examination of the patient's organic acid profile revealed large peaks of pyroglutamic acid (5-oxoproline) and paracetamol. Termination of paracetamol use, administration of $\mathrm{N}$-acetylcysteine to replenish the glutathione stores and haemofiltration caused resolution of the patient's acidaemia. Subsequent testing of the infants urine revealed no further evidence of pyroglutamate.

Conclusions Pyroglutamic aciduria (5-oxoprolinuria) is usually reported in children in the context of inherited errors of metabolism. The transient form that we describe here, whilst reported in the adult population, has rarely been described in children. Various pharmaceutical interventions, most commonly paracetamol, in combination with severe sepsis have been linked with this transient phenomenon. When the lactate and ketone levels don't correlate with the anion gap, it is important to pursue further diagnostic testing as illustrated by this case. In addition, scrutiny of the child's medication may give a clue to the diagnosis.

\section{G383(P) TAKING A CHILD HOME TO DIE: THE CHALLENGES OF DISCHARGING A PATIENT HOME FOR PALLIATIVE CARE FROM THE PAEDIATRIC INTENSIVE CARE UNIT}

C Kanaris, R Yates, D Morgan. Paediatric Intensive Care Unit, Royal Manchester Children's Hospital, Manchester, UK

\subsection{6/archdischild-2015-308599.338}

The nature of paediatric intensive care medicine is such that when the battle with a disease process cannot be won, focus needs to be placed on achieving a dignified death. If the situation permits, families will often opt for a peaceful passing away in familiar surroundings, at home or in a hospice. We focus on the common practical considerations that need to be addressed in order to facilitate the return home; as well the necessary planning to conform to legal requirements following a child's expected death outside the hospital setting.

We look into what medications might be needed for symptom control and what the challenges of prescribing these in the community might be. We shall also look into the importance of assessing the home environment and social support in advance, as well as what the logistics of transporting the patient out of hospital are. Furthermore we touch upon who needs to be involved in the on-going medical care of the child, as well as possible contingency plans, should the child survive at home.

Finally we shall explore what the role of the intensive care physician is in facilitating what is required by law; including confirmation of death, discussions with the coroner, and transport to the mortuary. Good, advance planning enables clinicians to minimise the invasion of the family's privacy during these delicate moments, and helps make a child's death as dignified as possible.

\section{G384(P) A QUALITY IMPROVEMENT (QI) PROJECT TO INCREASE THE NUMBER OF VENTILATED DAYS BETWEEN UNPLANNED EXTUBATIONS WITHIN PAEDIATRIC INTENSIVE CARE - USING REAL TIME STATISTICAL MONITORING}

M Russell, J Alexander, L Hulme. Paediatric Intensive Care Unit, University Hospital of North Staffordshire, Stoke-on-Trent, UK

\subsection{6/archdischild-2015-308599.339}

Aims Unplanned extubation is an important quality issue. Quality surveillance showed that we were not achieving national targets for the number of ventilated days between unplanned extubations. A QI project was launched with the primary aim of increasing the number of ventilated days between unplanned extubations.

QI literature directed at unplanned extubations have used statistical methodology that detects significance at the end of a fixed time period. This does not allow continuous quality monitoring. "G type" charts are a type of statistical quality control chart that exhibit improved sensitivity over conventional statistical approaches when dealing with rare events.

Methods A Root cause analysis showed us that most unplanned extubations were secondary to the endotracheal tube (ETT) slipping through loose tapes in lightly sedated patients. A series of interventions (Table 1) were implemented. Following intervention implementation we performed continuous monitoring using a "g-type" chart (Figure 1).

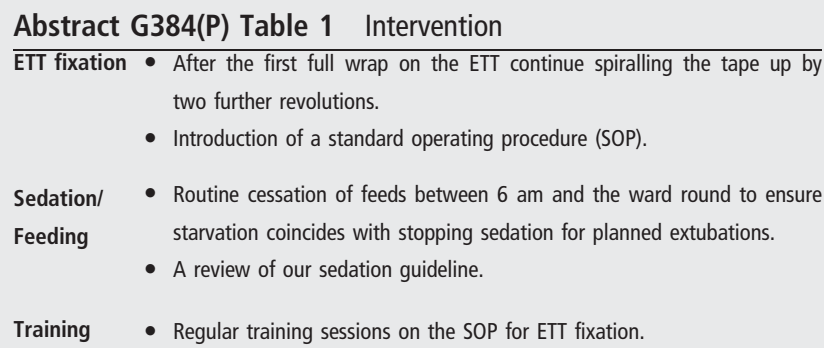

Results The mean number of ventilated days between unplanned extubations increased from 1 per 40.8 days to 1 per 250 days. G-chart 1 clearly illustrates that the mean number of ventilated 